\title{
Partizipatives Gesundheitsmanagement in der flexiblen Produktion: Entwicklung und Evaluation des teambezogenen Präventionskonzeptes SePIAR
}

\author{
Anja Gerlmaier ${ }^{1}$ \\ Angenommen: 9. November 2020 / Online publiziert: 26. November 2020 \\ (c) Der/die Autor(en) 2020, korrigierte Publikation 2021
}

\section{Zusammenfassung}

Seit kurzem existieren erste arbeitswissenschaftliche Erkenntnisse über gesundheitsbezogene Risiken und Gestaltungspotenziale bei digital vernetzten Produktionssystemen. Allerdings gibt es bisher kaum auf Arbeitsgestaltung abzielende Präventionsansätze, die sich für eine Stärkung der Gesundheitsressourcen in diesen von hohen Veränderungsdruck geprägten Arbeitskontexten als wirksam erwiesen haben.

Im Beitrag wird das Präventionskonzept SePIAR vorgestellt und über Ergebnisse einer Wirksamkeitsanalyse in acht Arbeitsbereichen aus der Produktion bzw. produktionsnahen Wissensarbeit berichtet. Das Verfahren richtet sich an Teams und ihre Führungskräfte und will diese befähigen, Stressursachen in ihrem Arbeitsbereich zu erkennen und kollektive Handlungsressourcen zu mobilisieren. Die Evaluation deutet auf positive Effekte im Hinblick auf Empowerment, Beteiligungserleben, Handlungskompetenz und Erschöpfungserleben hin.

\section{Praktische Relevanz}

Die durch das Arbeitsschutzgesetz geforderte Regulation psychischer Belastungen stellt betriebliche Akteure des Arbeitsund Gesundheitsschutzes in vernetzten Produktionssystemen vor besondere Herausforderungen. Dort bestehen erhebliche Präventionshemmnisse aufgrund von geringen Zeit- und Personalkapazitäten und eine sehr hohe Veränderungsdynamik bei der Belastungssituation. Klassische bedingungsbezogene Instrumente wie Gesundheitszirkel sind nicht darauf ausgelegt, zeitnah Ergebnisse zu liefern. Das Präventionskonzept SePIAR setzt hier auf den Aufbau kollektiver Gestaltungskompetenz unter Anwendung weitreichender Mitwirkungsmöglichkeiten von Teams und ihren Führungskräften. Das Verfahren bietet einen fruchtbaren Ansatz, durch den Aufbau lokaler Gestaltungskompetenz Hemmnisse bei der Umsetzung der Gefährdungsbeurteilung Psyche zu überwinden.

Schlüsselwörter Psychische Erschöpfung · Partizipation · Intervention · Evaluation · Gesundheitsmanagement

Dr. Anja Gerlmaier

anja.gerlmaier@uni-due.de

1 Institut Arbeit und Qualifikation, Universität Duisburg-Essen,

Forsthausweg 2, 47057 Duisburg, Deutschland 


\title{
Participatory health management in flexible production systems: Development and evaluation of the team-based stress management program SePIAR
}

\begin{abstract}
New findings in flexible production systems give us more information about specific job risks and resources in this organizational setting. Due to this, there is less evidence, which health promotion practices could be effective to reduce work-related stress in workers, administrative staff and their supervisors. In this paper a multi-modal resource and stress management program for teams in flexible production systems and its outcomes in 8 teams with qualified and low qualified workers are presented. Results show, that the participative approach had predominantly positive effects in enforstering team empowerment, self reported participation, proactive coping strategies and well-being mesured 6-8 month after the intervention.

\section{Practical Relevance}

Stress reduction in flexible production is an extraordinary challenge for organizational safety and health management. There are enormous organizational barriers according to low time and personal capacities and a high dynamic of change. Partizipative health approaches like health circles are limited in effectiveness because of long term intervention. The SePIAR program focuses on enhancing collective work design skills in teams. The program presents a fruitful approach to overcome some organizational obstacles in psychosocial risk assessment through initiating local work design activities.
\end{abstract}

Keywords Mental exhaustion · Participation - Intervention - Evaluation · Occupational health management

\section{Hintergrund und Zielstellung}

Die industrielle Fertigung in Deutschland ist in zunehmendem Maße in weltweit vernetzten Wertschöpfungsketten eingebunden. An den Produktionsstandorten führt diese Entwicklung zu erheblichen Umwälzungen der Arbeitsorganisation und des Technik- bzw. Personaleinsatzes, um innerhalb der global aufgestellten Wertschöpfungsketten flexibel und wettbewerbsfähig zu bleiben (Warning und Weber 2017).

Auf dem Shopfloor machen sich diese Veränderungen u.a. durch die zunehmende Nutzung von unternehmensübergreifenden Softwaresystemen (z.B. Enterprise Resource Planing Systeme [ERP]), durch den zunehmenden Einsatz von Robotik oder durch die Umsetzung agilerer Produktionsstrategien bemerkbar (mit schnellerem Produktwechsel bzw. kleineren Losgrößen). Diese Veränderungen bergen durchaus auch neue Chancen für eine menschengerechtere Gestaltung der Tätigkeiten: Der veränderte Technikeinsatz erhöht die Lernoptionen, kann die Aufgabenvielfalt erweitern und bietet durch Möglichkeiten der technischen Entkopplung eine Vergrößerung von persönlichen Zeitspielräumen (Falkenberg et al. 2020). Demgegenüber entstehen auch neue Belastungskonstellationen, die sich je nach Arbeitsbereich und Tätigkeit jedoch sehr ausdifferenzieren können (Diebig et al. 2018; Meyer et al. 2019; Ahlers 2018): Für Tätigkeiten im Bereich der Maschinenbedienung hat sich gezeigt, dass der Einsatz ganzheitlicher Produktionssysteme das Risiko von Leistungsüberwachung, Termindruck sowie einer Abnahme der Aufgabenvielfalt durch den Wegfall dispositiver Aufgaben begünstigt (Gerst 2011). Erweiterte Erreich- barkeitsanforderungen durch Kunden oder Lieferanten in anderen Zeitzonen erhöhen insbesondere bei operativen Führungskräften, Instandhaltungsteams und im technischen Vertrieb das Risiko von unzureichenden Erholungszeiten und von Abgrenzungsproblemen zwischen der Arbeit und anderen Lebensbereichen (Gerlmaier 2018a). In den der Produktion vor- und nachgelagerten Dienstleistungsbereichen (z.B. technisches Projektmanagement, Controlling) berichten die Beschäftigten wiederum von einer Zunahme des Zeit- und Leistungsdrucks infolge von parallel zu bearbeitenden Aufträgen. Diese Belastungssituation verschärft sich oft durch den Einsatz kundenseitig vorgegebener, aber gestaltungsbedürftiger ERP-Systeme, die zu ungeplanten Zusatzaufwänden durch inkonsistente Daten und eine hohe Störungsanfälligkeit beitragen (Falkenberg et al. 2020; Maier et al. 2015).

Für den betrieblichen Arbeits- und Gesundheitsschutz ergibt sich aufgrund der beschriebenen neuartigen Belastungskonstellationen ein Handlungsdruck in zwei Richtungen: Zum einen können die beschriebenen psycho-sozialen Belastungen vermittelt über krankheitsbedingte Fehlzeiten und Fluktuationen die Lieferzuverlässigkeit und damit den wirtschaftlichen Erfolg von Unternehmen beeinträchtigen. Zum anderen sind Arbeitgeber nach $\S 5$ Abs. 1 ArbSchG verpflichtet, arbeitsbedingte psychische Belastungen zu erfassen und beurteilen sowie Maßnahmen zu deren Optimierung zu entwickeln, umzusetzen und auf ihre Wirksamkeit zu überprüfen. Hierbei ist weitgehend unklar, inwiefern bestehende Checklisten und Instrumente zur Gefährdungsbeurteilung bzw. Gestaltungsprinzipien bei der Beanspruchungsoptimierung geeignet sind, diese neuartigen Belas- 
tungskonstellationen hinreichend zu erfassen (Schaper et al. 2020; Diebig et al. 2018).

Ziel des Beitrags ist es vor diesem Hintergrund zu überprüfen, ob durch den Aufbau von kollektiver Gestaltungskompetenz auf Ebene der Teams eine Stärkung der Gesundheitsressourcen von Beschäftigten und ihren operativen Führungskräften erzielt werden kann. Im Beitrag wird zunächst ein Überblick über bestehende Präventionsinstrumente und ihre Wirksamkeit unter besonderer Berücksichtigung flexibilisierter Arbeitssysteme gegeben. Dem schließt sich eine Beschreibung der Entwicklung und Evaluation des Präventionskonzeptes SePIAR an, das zur Gesundheitsstärkung in vernetzten Produktionssystemen entwickelt wurde.

\subsection{Gesundheitsinterventionen in flexiblen Arbeitssystemen}

Im Rahmen des betrieblichen Gesundheitsmanagements kann eine Stärkung der Mitarbeitergesundheit sowohl durch die Optimierung von Belastungen als auch durch einen Aufbau von psychischen, sozialen oder arbeitsorganisatorischen Ressourcen vollzogen werden. Belastungsoptimierungen können zum Beispiel durch ergonomische bzw. Personaleinsatzmaßnahmen erreicht werden (Stab und Schulz-Dadaczynski 2017). Zum Ressourcenaufbau haben sich Gesundheitsinterventionen bewährt, die auf die Förderung individueller Bewältigungskompetenz, der Verbesserung der sozialen Arbeitsbeziehungen, einer Erweiterung des Handlungsspielraums oder der Qualifikationsmöglichkeiten abzielen (Bamberg und Busch 2006). Aktuelle Studien zeigen, dass eine Mehrheit von Unternehmen betriebliche Veränderungsprozesse und die hieraus resultierenden psychischen Befindensbeeinträchtigungen durch verhaltenspräventive Interventionen $\mathrm{zu}$ begegnen versucht (Lechleiter und Purbs 2019). Die Maßnahmen stellen sportliche Aktivierung, Selbstachtsamkeit oder Resilienz in den Mittelpunkt der Intervention, um besser mit dem steigenden Veränderungsdruck umgehen zu können. Metaanalysen finden für diese individualzentrierten Präventionsverfahren moderate Effekte im Hinblick auf Stress oder Resilienz der Teilnehmenden (Barthelmes et al. 2020; Guillaumie et al. 2017). Geringe Teilnahmefrequenzen bei freiwilligen Angeboten sowie hohe vorzeitige Abbruchquoten - insbesondere beim Einsatz von digitalen Selbstlerntools - bedingen jedoch oft, dass auf Unternehmensebene kaum nachweisbare Effekte bei der Fehlzeitenminderung oder bei der Verbesserung des Wohlbefindens festgestellt werden können (Hammer et al. 2015; Richardson und Rothstein 2008). In den Meta-Analysen zur Bewertung von Stressinterventionen finden sich nur wenige verhältnisorientierte Interventionsansätze, da die Felduntersuchungen oft die geforderten Qualitätskriterien für die Aufnahme in die Meta-Analysen nicht erfüllen (Barthelmes et al. 2020).
Verhältnisbezogenen Maßnahmen wird insbesondere dann eine hohe Wirksamkeit zugesprochen, wenn diese Interessen der Zielgruppen und die betrieblichen Settings bei der Planung und Umsetzung berücksichtigen (Leider et al. 2015; Bamberg und Busch 2006).

Im Rahmen des betrieblichen Gesundheitsmanagements werden zur Erfüllung dieser Kriterien häufig ZirkelarbeitKonzepte oder Projektgruppen genutzt (z. B. im Nachgang von Gefährdungsbeurteilungen oder Gesundheitsbefragungen). Diese arbeiten in der Regel auf Basis von betrieblichen Gesundheitsdaten in Arbeitsgruppen über mehrere Sitzungen hinweg als „Experten vor Ort" Gestaltungsideen aus, die dann in betrieblichen Arbeits- und Gesundheitsschutzgremien vorgestellt und von betrieblichen Entscheidern im Hinblick auf ihre Realisierbarkeit bewertet werden (Stummer et al. 2008). Reviews zeigen eine gemischte Evidenz (Aust und Ducki 2004). Gesundheitseffekte finden sich eher bei Teilnehmenden bzw. in Interventionen, bei denen Beschäftigte erhebliche Mitwirkungsmöglichkeiten hatten (Kampe et al. 2020; Elke 2018; Semmer 2011; Wellendorf et al. 2001; Heaney et al. 1995). Umsetzungshemmnisse treten oft dort auf, wo das obere Management wenig Commitment bei der Umsetzung zeigt (Busch 2010), Gestaltungsprozesse innerhalb der Strukturen und Abläufe nicht ausreichend verankert sind (Stummer et al. 2008), sich Führungskräfte mittlerer und unterer Ebenen nicht ausreichend informiert und beteiligt fühlen (Banutu-Gomez 2015), Personalengpässe die regelmäßige Teilnahme an Zirkelgruppen beeinträchtigen oder sich der Zirkel aus weisungsbefugten und Untergebenen zusammensetzt (Aust und Ducki 2004).

Busch (2010) betont darüber hinaus, dass eine nachhaltige Stärkung der Gesundheitsressourcen eher bei natürlichen Teams erreicht werden kann, weil kollektive Lernprozesse unter Berücksichtigung persönlicher Interessen der Zielgruppen dort wahrscheinlicher sind als bei Zirkelgruppen aus verschiedenen Arbeitsbereichen und -ebenen (vgl. auch Lee et al. 2014; Ducki 2009). Evaluationsergebnisse aus dem ReSuM-Projekt (Busch 2010) zeigten in einer Stichprobe mit überwiegend gering qualifizierten Beschäftigten mit Migrationshintergrund, dass Teamkonzepte zur Stärkung von Gesundheitsressourcen sehr wirkungsvoll sind, wenn sie die Teamarbeitsqualität verbessern können.

Für Beschäftigtengruppen mit einem hohen technologischen Veränderungsdruck bestehen arbeitswissenschaftliche Gestaltungsempfehlungen aktuell darin, die Freiheitsgrade der dort Beschäftigten zu erhöhen, um die durch den Veränderungsdruck bedingten Belastungen abzupuffern (Meyer et al. 2019). Bisher existieren für diese Zielgruppen keine kontextsensitiven bedingungsbezogenen Präventionskonzepte, die diesen Gestaltungsempfehlungen gerecht werden, obwohl dort ein erheblicher präventiver Handlungsbedarf existiert. Zur Überwindung dieser Forschungs- 
und Gestaltungslücke wurde im Rahmen des BMBF-geförderten Vorhabens „InGeMo“ (siehe Hinweis am Ende des Textes) ein theoretisch fundiertes Präventionskonzept auf Basis von Methoden der Aktionsforschung entwickelt. Im nachfolgenden Beitrag wird das multi-methodale, auf umfangreiche Mitwirkung setzende Konzept vorgestellt und über die Wirkungen bei Produktionsteams mit niedrig qualifizierten und Facharbeiter*innen sowie Teams aus produktionsnahen Dienstleistungsbereichen berichtet.

\section{Methodisches Konzept und Vorgehensweise}

\subsection{Konzeptioneller Ansatz von SePIAR}

Im BMBF-geförderten Vorhaben InGeMo wurde das Ziel verfolgt, gemeinsam mit mehreren größeren und kleineren Kooperationsunternehmen aus der Elektro- und Metallindustrie ein beteiligungsorientiertes Präventionskonzept zur Stärkung der psycho-sozialen Gesundheitsressourcen in flexiblen Produktionsregimen zu entwickeln. Mit dem SePIAR-Verfahren wurde ein Vorgehensmodell konzipiert, mit dem auf lokaler Ebene Arbeitsgestaltungskompetenz von Führungskräften und ihren Teams zur Gesundheitsprävention aufgebaut werden kann. Ziel von SePIAR ist es, durch die Förderung von arbeitsbezogener Handlungskompetenz Mitarbeitende und ihre Führungskräfte zu befähigen, gesundheitliche Risiken und mögliche Gestaltungspotenziale entsprechend des Ansatzes der organisationalen Gestaltungskompetenz (Gerlmaier 2018b) frühzeitig zu erkennen und Präventionslösungen eigenständig zu entwickeln bzw. umzusetzen.

Der Begriff SePIAR steht dabei für ein stufenweises Vorgehen, das aus einer Stress-Sensibilisierung und dem Aufbau arbeitswissenschaftlich fundierten Gestaltungswissens (Se), einer Priorisierung relevanter Problemschwerpunkte und einer Entwicklung von Theorien über ihre Ursachen $(P)$, der Initiierung von Gestaltungsideen (I), der Aktivierung von Führungskräften und Mitarbeitenden zur Umsetzung (A) und der gemeinsamen Festlegung vom Umsetzungsmaßnahmen sowie einer Reflexion des Gesamtteams (R) besteht. Das Vorgehensmodell lehnt sich damit konzeptionell an den Lernzyklus von Argyris und Schön (1978) an.

Das SePIAR-Workshop-Konzept basiert auf zunächst parallel verlaufenden Mitarbeiter- und FührungskräfteWorkshops eines Arbeitsbereiches. Die dort erarbeiteten Handlungsbedarfe und Gestaltungsvorschläge werden zum Ende der Workshop-Reihe in einer gemeinsamen Sitzung mit Beschäftigten, operativen Führungskräften und Wissenschaftlerinnen reflektiert und gemeinsame Vereinbarungen zu Umsetzungsmaßnahmen getroffen. Anders als bei Zir-

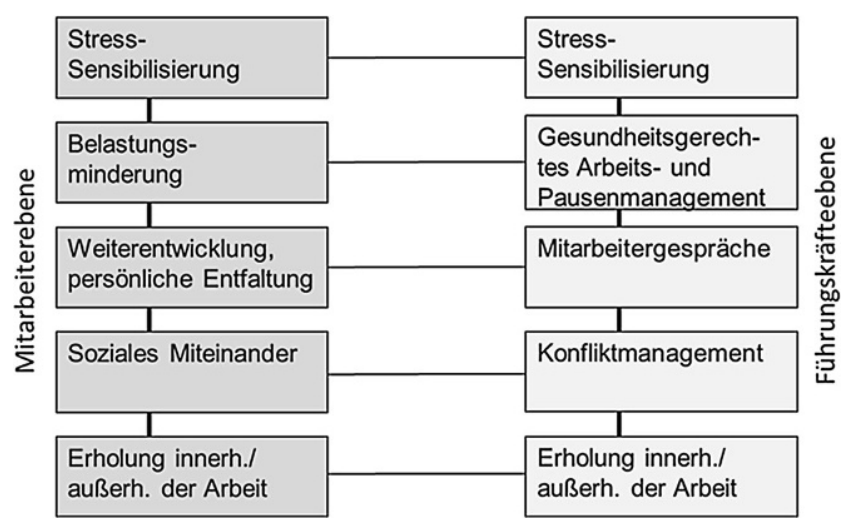

Abb. 1 Inhalte des Workshopkonzepts

Fig. 1 Contents of the workshop program

kelkonzepten nehmen an den Workshops nicht einzelne Beschäftigte als Stellvertreter von Arbeitsbereichen oder Führungsebenen teil. Bei dem Workshop-Konzept wird der Versuch unternommen, möglichst viele in einem Arbeitsbereich tätige Beschäftigte und Führungskräfte an den Workshops zu beteiligen.

Die Workshopgruppen können nach der Sensibilisierungsphase verschiedene Themen zur Stressprävention auswählen, für die sie im Team Handlungsbedarf sehen (siehe Abb. 1). Folgende Module können von den Teams ausgewählt werden: Arbeitsbelastungen vermindern, soziales Miteinander im Team, Erholung innerhalb und außerhalb der Arbeit, persönliche Weiterentwicklung. Die Führungskräfte können zudem Module zum Thema Konfliktmanagement, gesundheitsgerechte Arbeitsgestaltung, Erholung innerhalb und außerhalb der Arbeit sowie zu Mitarbeitergesprächen wählen. Das Konzept folgt damit Grundprinzipien der Aktionsforschung: Die Zielgruppen beteiligen sich freiwillig an der Gesundheitsintervention. Sie werden im Rahmen der Workshops sowohl an der Problemfindung und der notwendigen Bildung einer sogenannten „lokalen Theorie“ über die Ursachen beteiligt d.h. einer geteilten Vorstellung über das Zustandekommen des Problems -, als auch an der Entwicklung, Planung und Umsetzung von Maßnahmen sowie der Evaluation der Ergebnisse (Wright et al. 2013).

Neben einer weitestgehenden Beteiligung bestanden weitere Gestaltungskriterien für das Workshopkonzept darin, ein für flexible Produktionsregimes praktikables Instrumentarium zu entwickeln. Die Workshopmodule für Beschäftigte und Führungskräfte dauern 2,5h und können vor oder nach Arbeitsschichten durchgeführt werden. Die Anzahl der Workshop-Module kann von den Workshop-Teilnehmenden frei gewählt werden, bis zu maximal sechs Modulen (eine Sensibilisierungseinheit, bis zu vier Themenmodule und ein Reflexionsmodul). Anders als bei Gesundheitszirkeln können in Workshopverlauf entste- 
hende Fragen oder Gestaltungsvorschläge zwischen den Workshopgruppen der Mitarbeitenden und Führungskräfte zeitnah ausgetauscht werden, sofern dies von der jeweiligen Workshopgruppe gewünscht ist. So können innerhalb eines kurzen Zeitraums sowohl Handlungsschwerpunkte für die Prävention identifiziert als auch Umsetzungsmaßnahmen durchgeführt werden. Bei der Konzeption der Workshopmaterialien wurde auf eine einfache Sprache geachtet.

\section{2 Überprüfung der Wirksamkeit: Fragestellung}

Wie der Interventionsansatz, so orientierte sich auch die Wirksamkeitsüberprüfung an Kriterien partizipativer Forschungsansätze: Diese gehen davon aus, dass durch die intensive Beteiligung der Zielgruppe ihre Kompetenzen im Umgang mit dem lokalen Problem sowie ihr Engagement gesteigert werden können, sich an der Erarbeitung und Umsetzung von Gestaltungslösungen zu beteiligen. Durch den hohen Grad an Mitwirkung wird ein Aufbau sowohl von personalen Ressourcen wie eine Verbesserung bei der Selbstwirksamkeit und der Kontrollwahrnehmung erwartet. Sofern Gestaltungsmaßnahmen umgesetzt werden, kann eine Beeinflussung der Gesundheit auch über den Aufbau sozialer bzw. arbeitsorganisatorischer Ressourcen (z.B. Ergonomie, Verbesserung der Ablauforganisation oder der Kommunikation) erzielt werden (Bedingungsbezogene Veränderungen).

Aufbauend auf diesen Zielkriterien ergaben sich für die Wirksamkeitsbewertung des SePIAR-Verfahrens in Bereichen der Produktion bzw. produktionsnahen Dienstleistungen folgende Fragestellungen:

- In welchem Ausmaß gelingt es den Untersuchungsteams, auf Basis zuvor identifizierter Belastungssituationen von Teammitgliedern und ihren Führungskräften gemeinsam getragene Gestaltungsmaßnahmen umzusetzen?

- Können durch die Workshops Verbesserungen bei der Gestaltungskompetenz (Umgang mit Belastungen und Stress im Team) erzielt werden?

- Inwiefern kann durch die Workshops eine Verbesserung der teambezogenen Partizipationsmöglichkeiten (z.B. Beteiligung an der Gestaltung des Arbeitsplatzes, selbstbestimmtere Arbeitszeiteinteilung) erreicht werden?

- Inwieweit beeinflussen die Workshops das subjektive Wohlbefinden sechs Monate nach Abschluss der Maßnahme?

- Inwieweit wirken sich unterschiedliche betriebliche Settings der Untersuchungsteams (Qualifikations- bzw. Tätigkeitsunterschiede) auf die Wirksamkeit der Workshops aus?

\subsection{Untersuchungsdesign und Teilnehmende}

Im Rahmen des InGeMo-Projektes wurde das WorkshopKonzept in zwölf Teams aus Produktions- und Dienstleistungsbereichen von fünf Unternehmen der Elektro- und Metallindustrie durchgeführt. Bei den Kooperationsunternehmen handelte es sich um vier Zulieferunternehmen und einen Endhersteller aus der Automobilindustrie. In die Evaluation flossen Daten aus acht Teams ein, in vier Teams konnte aufgrund von Restrukturierungen keine Evaluation mehr durchgeführt werden. Bei den an der Evaluation beteiligten Teams handelte es sich um zwei Arbeitsbereiche mit überwiegend angelernten Maschinenbedienern (Mitarbeiteranzahl zwischen 24 und 185 Beschäftigten) sowie zwei Bereiche aus dem Werkzeugbau mit überwiegend qualifizierten Facharbeiter*innen (mit zwischen 31 und 114 Beschäftigten). Weitere vier Teams stammten aus unterschiedlichen Bereichen produktionsnaher Wissensarbeit (Controlling, technisches Projektmanagement, Produkttest, Einkauf). Die Teamgrößen lagen hier zwischen 24 und 60 Beschäftigten. Aus einem mittelständischen Automobilzulieferer und einem Stahl produzierenden Großunternehmen mit 4000 Beschäftigten nahmen jeweils ein Arbeitsbereich mit angelernten Beschäftigten, Facharbeiter*innen und produktionsnahen Dienstleistungstätigkeiten (technisches und kaufmännisches Auftragsmanagement) teil. Je ein weiteres Untersuchungsteam stammte aus dem Produkttest eines mittelständischen Motorenentwicklers sowie dem Controlling eines Automobilkonzerns.

Zur Wirksamkeitsmessung wurden verschiedene Datenquellen genutzt: Anhand schriftlicher Befragungen der Teammitglieder und ihrer Führungskräfte vor den Workshops und 6-8 Monate nach Abschluss der Workshops wurden Veränderungen bei Gesundheitsparametern, der Arbeitsgestaltungskompetenz und Facetten der erlebten Arbeitssituation (z.B. Partizipationsmöglichkeiten) ermittelt. Nach Abschluss der Maßnahmen wurden die Teammitglieder und Führungskräfte darüber hinaus gebeten, den Grad des Umsetzungserfolges für arbeitsorganisatorische Veränderungen zu beurteilen. Als weiteres Erfolgskriterium wurde herangezogen, wie viele Maßnahmen auf Basis der Workshops 6-8 Monate nach dem Workshop-Ende zur Umsetzung gekommen waren.

An der schriftlichen Vorher-Befragung nahmen 622 Personen teil, bei der Nachher-Befragung waren es 302 Personen. In die Evaluationsstudie zur Wirksamkeitsüberprüfung des Präventionskonzeptes flossen letztlich die Daten von 174 Teilnehmenden aus acht Arbeitsbereichen ein, für die Daten von Messzeitpunkt $T_{1}$ und $T_{2}$ anhand von Kennungen eindeutig einer Person zugeordnet werden konnten. Diese Daten wurden anhand von abhängigen T-Tests mithilfe des Statistikprogramms SPSS Release 26 ausgewertet. Das Verfahren fand Anwendung, weil mit Döring und Bortz (2016, 
S. 245) davon ausgegangen wird, dass die im Fragebogen verwendeten Abstufungen mit fünf Antwortmöglichkeiten (,stimme voll und ganz zu“ bis „stimme überhaupt nicht zu“) zumindest als annähernd äquidistant zu bewerten sind (vgl. auch Rohrmann 1978).

Von den 174 Befragten waren 87,4\% männlich. In den vier Teams aus dem Bereich der produktionsnahen Wissensarbeit lag der Frauenanteil mit 22,8\% höher als in den beiden Bereichen aus der Produktion (4,2\% bei Anlerntätigkeiten, 4,2\% bei Facharbeit). Erwartungsgemäß unterschieden sich die drei Tätigkeitsgruppen auch im Hinblick auf das Bildungsniveau: bei den Teams mit Anlerntätigkeiten dominierte ein einfacher Schulabschluss (91,7\%), bei den Teams mit überwiegender Facharbeit gaben $67,1 \%$ einen einfachen und 31,4\% einen mittleren Schulabschluss an. Bei den Teams mit Wissensarbeit gaben 20,5\% einen einfachen, 43,6\% einen mittleren und 35,9\% einen höheren Schulabschluss an. Die Befragungen zu Messzeitpunkt $\mathrm{T}_{1}$ und $\mathrm{T}_{2}$ fanden in den Räumlichkeiten der jeweiligen Kooperationsbetriebe und während der regulären Arbeitszeiten, meist kurz vor Beginn bzw. Ende der Schichten statt. Während der Befragungen der Teams aus dem Bereich der Produktion wurde gewährleistet, dass entweder eine Wissenschaftlerin des Forschungsprojektes oder Vertreter*innen des Betriebsrats bzw. Sicherheitsbeauftragte den Teilnehmenden beim Ausfüllen des Fragebogens behilflich sein konnten.

\subsection{Evaluationsinstrumente}

Als Kriterien für die Evaluation wurden Veränderungen beim subjektiven Wohlbefinden, der erlebten Beteiligung und der Arbeitsgestaltungskompetenz sowie das Ausmaß des Empowerments innerhalb der Teams betrachtet.

Zur Messung der ersten drei Kriterien dienten Ergebnisse der schriftlichen Vorher- und Nachher-Befragungen. In die Befragungen wurden auch Teammitglieder eingeschlossen, die nicht an den Workshops teilgenommen hatten. Zur Messung möglicher Veränderungen des psycho-sozialen Wohlbefindens wurde die Skala „Erschöpfung“ der deutschen Version des Maslach-Burnout-Inventory verwendet (Büssing und Perrar 1992). Die Skala, bestehend aus fünf Items, erfragt mithilfe einer fünf-stufigen Skala von 1 (,trifft völ- lig zu“) bis 5 (,trifft gar nicht zu“) verschiedene Facetten psychischer Erschöpfung ab. Die Befragten können hier beispielsweise angeben, in welchem Maß sie sich am Ende eines Arbeitstages verbraucht fühlen. Zur Messung des Kriteriums „Partizipation“ wurde die gleichnamige Skala des Instruments zur stressbezogenen Arbeitsanalyse (ISTA) von Semmer et al. (1999) verwendet. Die Befragten können dort ankreuzen, wie viel Einfluss sie auf verschiedene Aspekte ihrer Arbeit haben. Beispiel-Item: „Wie viel Einfluss haben Sie auf die Ausgestaltung Ihres Arbeitsplatzes?“ Hierbei können sie zwischen fünf Stufen der Beteiligung von 1 („Ich habe keinerlei Einfluss"), 2 („Ich werde nur informiert"), 3 (,Ich kann Vorschläge machen“) bis hin zu 4 (,Ich werde an der Entscheidung beteiligt") sowie 5 (,Ich habe großen Einfluss.“) wählen.

Zur Messung der Gestaltungskompetenz lag kein standardisiertes und validiertes Verfahren vor, weswegen hier eine Neukonstruktion notwendig war. Mit der Skala „Arbeitsbezogene Handlungskompetenz" sollten proaktive Verhaltensweisen erhoben werden, die Arbeitende zur Reduktion von psychischer Belastung und Stress nutzen können. Hierzu fanden zunächst umfangreiche Literaturanalysen statt. Im Anschluss daran erfolgte die Formulierung von acht Items zu Stress mindernden Verhaltensweisen. Diese Items wurden 25 betrieblichen und wissenschaftlichen Expert*innen vorgelegt, um diese sprachlich bzw. inhaltlich zu überprüfen. Nach einem faktorenanalytischen Test auf Basis einer Stichprobe von 622 Befragten wurde die Skala zur Optimierung der Konsistenz auf eine 5-Item-Lösung komprimiert. Beispiel Item: „Ich spreche regelmäßig mit Kolleg*innen oder der/dem Vorgesetzten darüber, wie ich/wir unsere Arbeit besser gestalten und Belastungen vermindern können“. Als Antwortformat diente eine fünfstufige LikertSkala von 1 (,trifft völlig zu“) bis 5 (,trifft gar nicht zu“). Die Skalen wiesen eine gute bis befriedigende interne Konsistenz (Cronbachs Alpha) auf (Tab. 1).

Für die Messung des Ausmaßes des innerhalb der Workshops erzeugten Empowerments wurden Dokumentationen aus den Mitarbeiter- und Führungskräfte-Workshops herangezogen. Dabei wurde davon ausgegangen, dass die Anzahl umgesetzter Gestaltungsmaßnahmen in positivem Zusammenhang mit dem Empowerment in den Teams steht. Als Kriterium diente die Anzahl der innerhalb der Umsetzungs-

Tab. 1 Reliabilität der Evaluationsskalen auf Basis der Vorher-Befragung (Quelle: eigene Daten)

Table 1 Reliability of evaluation scales based on the pretest data (source: own data)

\begin{tabular}{llll}
\hline Skala & Anzahl Items & Reliabilität & Beispiel-Item \\
\hline Psychische Erschöpfung & 5 & 0,86 & $\begin{array}{l}\text { Ich fühle mich wieder müde, wenn ich aufstehe und den nächsten Arbeitstag vor } \\
\text { mir habe } \\
\text { Arbeitsbezogene Hand- }\end{array}$ \\
$\begin{array}{l}\text { lungskompetenz } \\
\text { Partizipation }\end{array}$ & 7 & 0,67 & $\begin{array}{l}\text { Bei der Planung meiner Arbeit achte ich darauf, Zeitpuffer für Unvorhergesehe- } \\
\text { nes einzuplanen, wenn dies arbeitsorganisatorisch möglich ist } \\
\text { Wie viel Einfluss haben Sie bei der Planung der Arbeitszeit (Schichten und } \\
\text { Überstunden)? }\end{array}$ \\
\hline
\end{tabular}


und Reflexionsphase von Führungskräften und Mitarbeitenden vereinbarten und realisierten Gestaltungsmaßnahmen. In die Analyse gingen nur Gestaltungsmaßnahmen ein, die von beiden Gruppen in der Evaluationsphase übereinstimmend als umgesetzt deklariert wurden. Mittels der Methode der axialen Kodierung erfolgte in einem weiteren Schritt eine induktive Kategorisierung der Umsetzungsmaßnahmen. Diese wurden auf Verbindungen bzw. Unterschiede des präventiven Ansatzpunktes hin untersucht und ein Kategoriensystem mit 8 Oberbegriffen (z. B. Förderung der Erholungskompetenz) entwickelt (vgl. Ellinger 2003). Durch zwei wissenschaftliche Mitarbeitende des Forschungsprojektes erfolgte sodann eine unabhängige Zuordnung der Umsetzungsmaßnahmen zu den Oberkategorien. 78 Umsetzungsmaßnahmen wurden von den Beurteilenden der gleichen Kategorie zugeordnet. Die verbleibenden vier indifferenten Zuordnungen konnten in einem gemeinsamen Reflexionsschritt aufgelöst und die Gestaltungsmaßnahmen zugeordnet werden.

\section{Ergebnisse}

\subsection{Empowerment}

Innerhalb der Workshops wurden durch die beteiligten Beschäftigten und ihre Führungskräfte zahlreiche Präventionsmaßnahmen zum Aufbau von Gesundheit stärkenden Ressourcen entwickelt und innerhalb der Evaluationsphase von acht Monaten umgesetzt. Insgesamt konnten 82 Gestaltungsmaßnahmen verwirklicht werden, wobei die Anzahl der Gestaltungsmaßnahmen in Abhängigkeit vom Tätigkeitsbereich stark variierte. Mit im Durchschnitt elf bzw. zwölf durchgeführten Maßnahmen wiesen die Teams aus dem Bereich der Facharbeit und produktionsnahen Wissensarbeit doppelt so viele erfolgreiche Gestaltungsmaßnahmen auf wie die beiden Teams mit überwiegender Anlerntätigkeit (durchschnittlich sechs Maßnahmen). Bei beiden Teams aus dem Bereich der Maschinenbedienung fiel auf, dass in den Abschluss-Workshops bei der Entscheidung über die Umsetzung von Gestaltungsmaßnahmen kaum Gestaltungsvorschläge aus den BeschäftigtenWorkshops Berücksichtigung fanden, obwohl diese aus

Tab. 2 Umsetzungsmaßnahmen in den untersuchten Bereichen $(\mathrm{A}=$ Anlerntätigkeit, $\mathrm{F}=$ Facharbeit, W=Wissensarbeit, Quelle: eigene Daten) Table 2 Intervention results, divided in low qualified $(=\mathrm{A})$, qualified $(=\mathrm{F})$ and administrative teams (= W, source: own data)

\begin{tabular}{|c|c|c|c|c|}
\hline Handl. feld & Umsetzungsmaßnahme & A & $\mathrm{F}$ & $\mathrm{W}$ \\
\hline \multirow[t]{3}{*}{$\overline{\text { Erholungskompetenz }}$} & Einführung von Pausenräumen & - & 2 & 1 \\
\hline & Individuelles Erholungsmanagement & 1 & 2 & 3 \\
\hline & Entwicklung von Pausenritualen & 1 & 2 & 2 \\
\hline \multirow[t]{4}{*}{ Transparenz } & Rollenklärung & - & - & 2 \\
\hline & $\begin{array}{l}\text { Durchführung regelmäßiger Gruppensitzungen (Aufwertung durch Anwesenheit } \\
\text { des Vorgesetzten) }\end{array}$ & 2 & 2 & 2 \\
\hline & Einführung abteilungsübergreifender Regeltermine zum Wissensaustausch & - & 2 & 2 \\
\hline & Belastungskommunikation in Teamsitzungen & - & 2 & 1 \\
\hline \multirow[t]{5}{*}{ Zeitsouveränität } & Einführung von Block-Zeiten für konzentrationsintensive Aufgaben & 1 & 1 & 4 \\
\hline & Einführung von Ruhe-Arbeitsplätzen & - & 1 & 2 \\
\hline & Vereinbarungen zu Kurzpausen & 1 & 1 & 3 \\
\hline & Gewährung von Home Office-Anteilen & - & - & 3 \\
\hline & Einführung flexibler Mittagspausen & - & 1 & 1 \\
\hline \multirow[t]{2}{*}{$\begin{array}{l}\text { Kompetenzent- } \\
\text { wicklung (tech.) }\end{array}$} & $\begin{array}{l}\text { Einführung von Mitarbeiter-Entwicklungsgespr. mit verbindlicher Festlegung des } \\
\text { Qualifizierungsbedarfs }\end{array}$ & - & 2 & 3 \\
\hline & Unterweisungen/Trainings zu neuen Arbeitsmaterialien und Prozessen & 2 & 1 & 1 \\
\hline \multirow[t]{2}{*}{ Kapazitätsbemessung } & Optimierung der Personalkapazitätsplanung zur Vermeidung von Mehrarbeit & - & 1 & 2 \\
\hline & $\begin{array}{l}\text { Einführung entlastender Kooperationsformen (z. B. Arbeitstandems, Vertretungsre- } \\
\text { gelungen) }\end{array}$ & - & - & 3 \\
\hline \multirow[t]{2}{*}{ Sozialklima } & Führungskräftecoaching zu gesundheitsgerechter Führung & 1 & 1 & 2 \\
\hline & Teamentwicklungsmaßnahmen & - & 1 & 2 \\
\hline \multirow[t]{2}{*}{ Handlungsspielraum } & Beteiligung der MA an Planung, Werkzeugauswahl, Arbeitsplatzgestaltung etc & - & 1 & 3 \\
\hline & Raumgestaltung, Beschaffung von ergonomischen Arbeitsmitteln & - & - & 3 \\
\hline \multirow{2}{*}{$\begin{array}{l}\text { Neuregelung } \\
\text { Erreichb./ } \\
\text { Mobilarbeit }\end{array}$} & Verstärkter Einsatz zur Vermeidung nicht finanzierter Dienstreisezeiten & - & - & 1 \\
\hline & Vereinbarungen zu Schichtübergaben & 1 & 1 & - \\
\hline Arbeitsteilung & Veränderte Arbeitsteilung & 2 & - & - \\
\hline
\end{tabular}


arbeitswissenschaftlicher Sicht sinnvoll waren (z.B. Verbesserung der Bedienfreundlichkeit bei einem eingesetzten Produktionsplanungs-Tool).

Die meisten umgesetzten Gestaltungsmaßnahmen zielten auf Erweiterungen von Zeit- und Handlungsspielräumen ab, etwa die Gewährung von Home-Office, freiere Zeiteinteilungen bei der Pausendurchführung oder mehr Beteiligung bei der Gestaltung des Arbeitsplatzes (19 Maßnahmen). Weitere häufig umgesetzte Gestaltungsmaßnahmen betrafen die Erhöhung der Transparenz von Arbeitsabläufen (15), z.B. die Einführung von teamübergreifenden Meetings zur Identifikation von stressauslösenden Störquellen. 14 Maßnahmen zielten auf eine Erhöhung der Erholungskompetenz ab (z.B. teambezogene Entspannungstrainings, Einführung von Kurzpausen). Wie in Tab. 2 dargestellt, setzten die Teams seltener Maßnahmen zur Kompetenzentwicklung bzw. zur Kapazitätsoptimierung (etwa die Einführung von Arbeitstandems) oder zur Verbesserung des Sozialklimas um.

\subsection{Beteiligungserleben}

Im Interventionszeitraum kam es insgesamt zu einem signifikanten Anstieg der erlebten Partizipationsmöglichkeiten (Mittelwert Vorher-Befragung 2,68, Mittelwert Nachher-Befragung 2,80, $\mathrm{T}=2,30, \mathrm{df}=173$, Signifikanz $p=0,02$, Effektstärke nach Cohen $d=0,17$ ). So sank beispielsweise beim Item „Einfluss auf die Pausengestaltung“ der Anteil derer, die keinerlei Einfluss haben oder nur informiert werden von $41,4 \%$ auf $30,8 \%$ (Abb. 2). Betrachtet man die Veränderungen bei den drei untersuchten Tätigkeitsgruppen genauer, so findet sich ein Anstieg bei den Teams aus dem Bereich Facharbeit und der produktionsnahen Wissensarbeit, während bei den Teams mit überwiegender Anlerntätigkeit eine leichte Abnahme zu beobachten ist (Abb. 3).

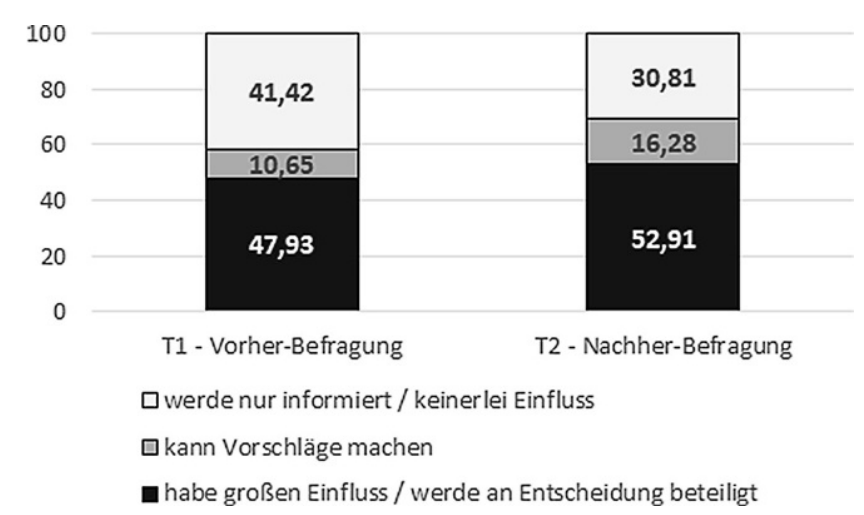

Abb. 2 Item „Einfluss auf die Pausengestaltung“ (Quelle: eigene Daten)

Fig. 2 Item „Influence on the design of breaks“ (source: own data)

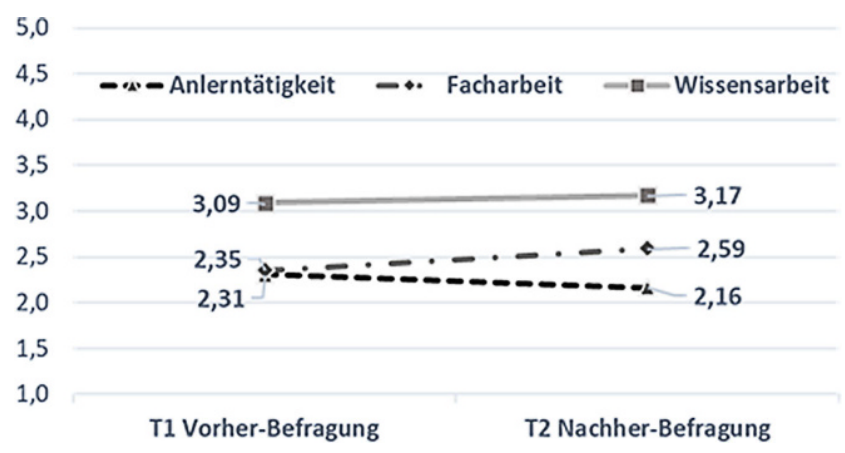

Abb. 3 Partizipationserleben differenziert nach Anlerntätigkeit, Facharbeit und produktionsnaher Wissensarbeit (Quelle: eigene Daten)

Fig. 3 self reported participation divided in low qualified, qualified and administrative teams (source: own data)

\subsection{Arbeitsbezogene Handlungskompetenz}

Zwischen den Messzeitpunkten $T_{1}$ und $T_{2}$ kann insgesamt ein tendenziell bedeutsamer Anstieg bei der Skala ,arbeitsbezogene Handlungskompetenz" beobachtet werden (Mittelwert Vorher-Befragung 3,06, Mittelwert Nachher-Befragung $3,16, \mathrm{~T}=1,90, \mathrm{df}=173$, Signifikanz $p=0,06$, Effektstärke $\mathrm{d}=0,14)$. Bei der Betrachtung der Tätigkeitsgruppen ergibt sich ein ähnlich differenziertes Bild wie bei den Partizipationsmöglichkeiten. Während die Kompetenzen zum Umgang mit Belastungen sich bei den Teams mit Facharbeit und Wissensarbeit deutlicher verbesserten, fand sich diese Entwicklung bei den Teams mit Geringqualifizierten nicht (Abb. 4).

\subsection{Psycho-soziales Wohlbefinden}

Im Zeitraum zwischen Vorher- und Nachher-Befragung kann auch eine Abnahme der erlebten psychischen Erschöpfung beobachtet werden (Mittelwert Vorher-Befragung 2,89, Mittelwert Nachher-Befragung 2,77; $\mathrm{T}=2,39$, $\mathrm{df}=173$, Signifikanz $p=0,02$, Effektstärke d=0,18). Fühl-

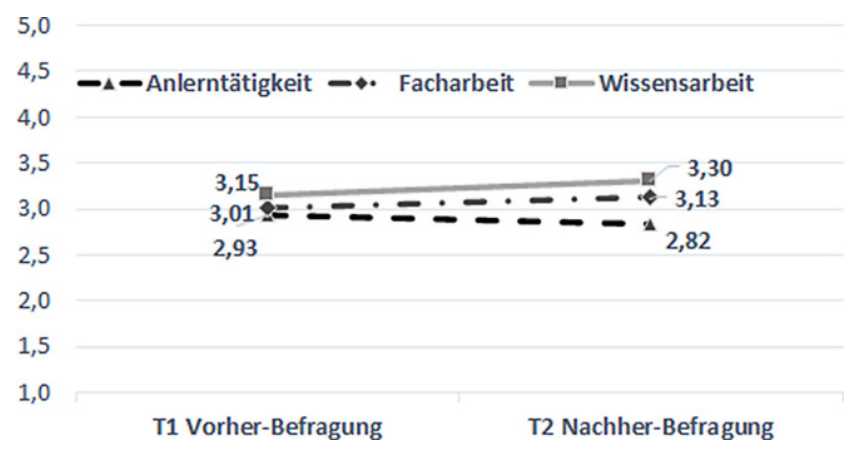

Abb. 4 Arbeitsbezogene Handlungskompetenz differenziert nach Anlerntätigkeit, Facharbeit und produktionsnaher Wissensarbeit (Quelle: eigene Daten)

Fig. 4 Work related proactive coping divided in low qualified, qualified and administrative teams (source: own data) 


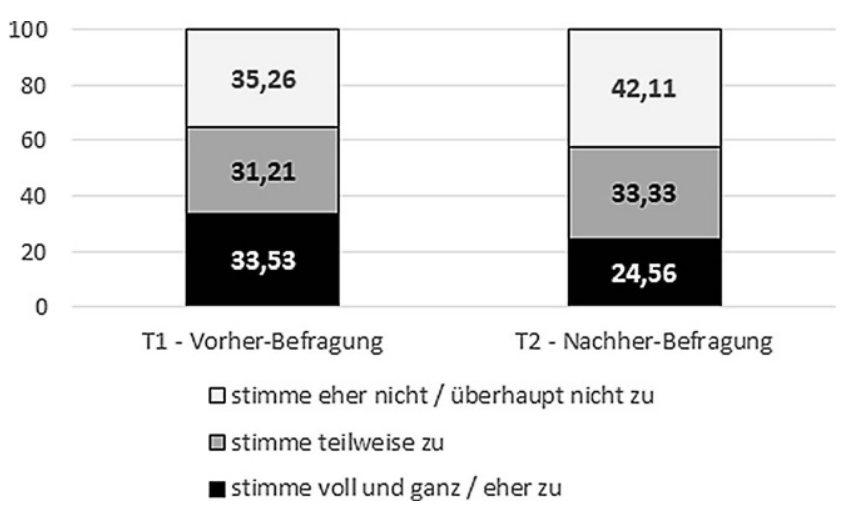

Abb. 5 Item „Ich fühle mich wieder müde, wenn ich aufstehe und den nächsten Arbeitstag vor mir habe.“ (Quelle: eigene Daten)

Fig. 5 Item „I feel tired again when I get up and have the next working day ahead of me“ (source: own data)

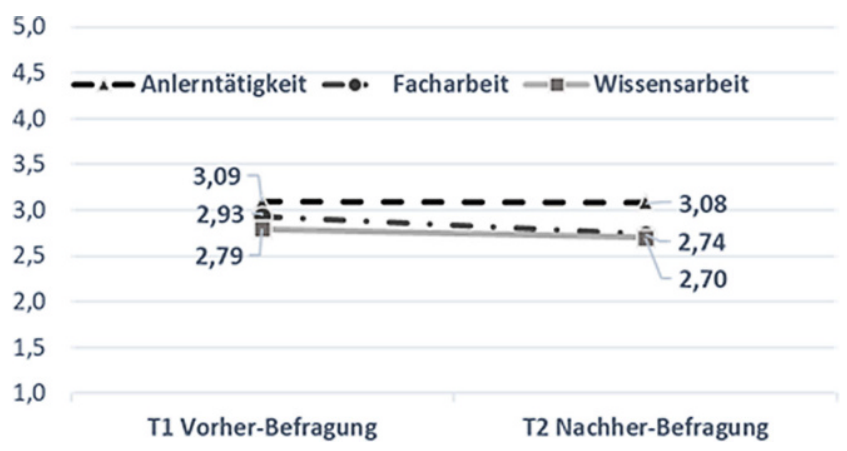

Abb. 6 Psychische Erschöpfung differenziert nach Anlerntätigkeit, Facharbeit und produktionsnaher Wissensarbeit (Quelle: eigene Daten)

Fig. 6 mental exhaustion divided in low qualified, qualified and administrative teams (source: own data)

ten sich beispielsweise vor der Durchführung der Workshops noch 33,5\% aller Befragten oft oder immer müde nach dem Aufstehen, so sind dies 6-8 Monate nach der Maßnahme nur noch 24,6\% (Abb. 5). Die deutlichsten Reduzierungen der erlebten Beanspruchung finden sich auch hier wieder bei Teams aus dem Bereich Facharbeit und Wissensarbeit (Abb. 6). Bei den Teams mit überwiegender Anlerntätigkeit ergab sich dagegen keine Veränderung.

\section{Diskussion}

Ein Ziel der vorliegenden Evaluationsstudie bestand darin zu überprüfen, ob durch den Einsatz des partizipationsorientierten SePIAR-Workshop-Konzepts für Teams Gesundheitsressourcen von Beschäftigten in hochflexiblen Produktionsregimes gestärkt werden können. Die Ergebnisse deuten darauf hin, dass mithilfe des Verfahrens in einem relativ kurzen Interventionszeitraum von zwischen sechs und zwölf Wochen das Arbeitsgestaltungs-Know-how, die subjektiv erlebten Partizipationsmöglichkeiten und das Aus- maß der psychischen Erschöpfung in der Mehrheit der untersuchten Teams günstig beeinflusst werden konnte.

Allerdings profitierten Teams aus den Kategorien Facharbeit und produktionsnahe Wissensarbeit mehr von dem Workshop-Konzept als die Niedrigqualifizierten. Dieses Phänomen ist auch in anderen Studien beschrieben worden und wird häufig mit Problemen bei der Interessenartikulation (Sprach- und Verständnisprobleme, Angst vor Sanktionen durch große Machtgefälle) erklärt (Busch 2010; Thompson et al. 2005). In unseren Workshops mit Niedrigqualifizierten fand sich jedoch ein anderes Erklärungsmuster: Beschäftigtenvorschläge beim gemeinsam angelegten Aushandlungsprozess mit den Vorgesetzten fanden in den Teams mit Geringqualifizierten deutlich weniger Berücksichtigung in der Umsetzung, obwohl diese von arbeitswissenschaftlich guter Qualität waren: In einem Untersuchungsfall ging die umsetzungsverantwortliche Führungskraft fast vollständig über die Gestaltungsvorschläge ihres Teams hinweg und setzte überwiegend Gestaltungsvorschläge aus dem Führungsteam um. Die resultierende Gestaltungsohnmacht der Workshop-Teilnehmer dürfte auch ein Erklärungsansatz für das verminderte Wohlbefinden und die als geringer wahrgenommenen Partizipationsmöglichkeiten in dieser Beschäftigtengruppe sein.

Bei der Implementierung des Workshop-Konzepts im Rahmen des betrieblichen Gesundheitsmanagements sollte daher zukünftig auf betriebliche Regelungen zu einem fairen Umgang mit Gestaltungsvorschlägen geachtet werden, um akteursspezifische Machtungleichheiten austarieren zu können. Der betriebliche Ausschuss für Arbeits- und Gesundheitsschutz kann hier als ein entsprechender institutioneller Anker fungieren, um bei konfliktären Gestaltungsansätzen auf arbeitswissenschaftlich fundierte Kompromisslösungen hinzuwirken.

\subsection{Wissenschaftliche Limitierung}

Bei der Bewertung der Befunde sind einige Limitierungen beim Studiendesign zu beachten. Das Präventionskonzept hat sich im Kontext flexibler Produktionsregime als weitgehend wirkungsvoll erwiesen, indem es zum Aufbau von kollektiven Arbeitsgestaltungskompetenzen auf lokaler Ebene beitragen kann. Ein randomisiertes Kontrollgruppen-Design wäre für die wissenschaftliche Bewertung der Wirkeffekte hilfreich gewesen, wurde jedoch durch die betrieblichen Kooperationspartner mit der Begründung betriebswirtschaftlich ungerechtfertigter Personalausfälle nicht unterstützt. Die abnehmende Akzeptanz betrieblicher Kooperationspartner für elaborierte Evaluationsdesigns bei Maßnahmen zum Gesundheitsmanagement dürfte mitverantwortlich sein, warum der Anteil verhältnisbezogener Präventionsmaßnahmen in wissenschaftlichen Reviews stetig abnimmt. Ein Lösungsansatz hierfür kann darin be- 
stehen, zur Evaluation von Maßnahmen zum betrieblichen Gesundheitsmanagement Wartegruppen-Designs zu nutzen, um hierdurch Kontrollgruppen in ähnlichen Arbeitskontexten zu erhalten. Dies setzt jedoch die Gewährung deutlich längerer Projektlaufzeiten voraus, als dies gegenwärtig vorzufinden ist.

\section{Hinweis}

Das Verbundprojekt „Initiative betriebliche Gestaltungskompetenz stärken - ein neues Präventionsmodell für Unternehmen und Beschäftigte“ (InGeMo) wurde gefördert vom Bundesministerium für Bildung und Forschung (BMBF), Förderkennzeichen 02L14A020, Laufzeit von April 2016 bis Juli 2019.

Funding Open Access funding enabled and organized by Projekt DEAL.

Open Access Dieser Artikel wird unter der Creative Commons Namensnennung 4.0 International Lizenz veröffentlicht, welche die Nutzung, Vervielfältigung, Bearbeitung, Verbreitung und Wiedergabe in jeglichem Medium und Format erlaubt, sofern Sie den/die ursprünglichen Autor(en) und die Quelle ordnungsgemäß nennen, einen Link zur Creative Commons Lizenz beifügen und angeben, ob Änderungen vorgenommen wurden.

Die in diesem Artikel enthaltenen Bilder und sonstiges Drittmaterial unterliegen ebenfalls der genannten Creative Commons Lizenz, sofern sich aus der Abbildungslegende nichts anderes ergibt. Sofern das betreffende Material nicht unter der genannten Creative Commons Lizenz steht und die betreffende Handlung nicht nach gesetzlichen Vorschriften erlaubt ist, ist für die oben aufgeführten Weiterverwendungen des Materials die Einwilligung des jeweiligen Rechteinhabers einzuholen.

Weitere Details zur Lizenz entnehmen Sie bitte der Lizenzinformation auf http://creativecommons.org/licenses/by/4.0/deed.de.

\section{Literatur}

Ahlers E (2018) Die Digitalisierung der Arbeit. Verbreitung und Einschätzung der Betriebsräte. WSI-Report Nr. 40. Hans-BöcklerStiftung, Düsseldorf

Argyris C, Schön DA (1978) Organizational learning: a theory of action perspective. Reading, Mass.

Aust B, Ducki A (2004) Comprehensive health promotion interventions at the workplace: experiences with health circles in Germany. J Occup Health Psychol 9(3):258-270

Bamberg E, Busch C (2006) Stressbezogene Interventionen in der Arbeitswelt. Z Arbeits Organisationspsychol 50(4):215-226

Banutu-Gomez M (2015) Understanding leadership and empowerment in the workplace. Eur Sci J 11(35):342-365

Barthelmes I, Bödeker W, Sörensen J, Kleinlercher KM, Odoy J (2020) Aktuelle Evidenzlage für Wirksamkeit und Nutzen arbeitsbezogener Gesundheitsförderung und Prävention. Iga.Report 40. IAG, Dresden

Busch C (2010) Teamarbeit und Gesundheit. In: Badura B, Schröder H, Klose J, Macco K (Hrsg) Fehlzeiten-Report 2009. Springer, Berlin

Büssing A, Perrar KM (1992) Die Messung von Burnout. Untersuchung einer deutschen Fassung des Maslach Burnout Inventory (MBI-D). Diagnostica 38(4):328-353
Diebig M, Jungmann F, Müller A, Wulf IC (2018) Inhalts- und prozessbezogene Anforderungen an die Gefährdungsbeurteilung psychischer Belastung im Kontext Industrie 4.0 - Eine qualitative Interviewstudie. Z Arbeits Organisationspsychol 62(2):53-67

Döring N, Bortz J (2016) Forschungsmethoden und Evaluation in den Sozial- und Humanwissenschaften, 5. Aufl. Springer, Berlin

Ducki A (2009) Stress und Ressourcenmanagement. In: Busch C, Roscher S, Ducki A, Kalytta T (Hrsg) Stressmanagment für Teams in Service, Gewerbe und Produktion - ein ressourcenorientiertes Trainingsmanual. Springer, Berlin, S 15-27 (Kapitel 1.2)

Elke G (2018) Präventionskultur: Erfolgsfaktor für nachhaltige Sicherheit und Gesundheit bei der Arbeit? In: Trimpop R, Kampe J, Bald M, Seliger I, Effenberger G (Hrsg) 20. Workshop Psychologie der Arbeitssicherheit und Gesundheit. Voneinander lernen und miteinander die Zukunft gestalten! Asanger, Kröning, S 12-20

Ellinger S (2003) Grounded Theory als methodischer Zugang für Werteforschung in der Lernbehindertenschule. In: Vernooij MA, Wittrock M (Hrsg) Beziehung (-s) Gestalten. BIS-Verlag, Oldenburg, S 95-116

Falkenberg J, Haipeter T, Krzywdzinski M, Kuhlmann M, Schietinger M, Virgillito A (2020) Digitalisierung in Industriebetrieben Auswirkungen auf Arbeit und Handlungsansätze für Betriebsräte. Forschungsförderung Report 6. Hans-Böckler Stiftung, Düsseldorf

Gerlmaier A (2018a) Gesund arbeiten im Zeitalter der Digitalisierung: Wo liegen die Arbeitsgestaltungspotenziale aus Sicht von Beschäftigten und Unternehmensvertretern? IAQ-Report Nr. 201804. Universität Duisburg-Essen Campus Duisburg, Institut Arbeit und Qualifikation, Duisburg

Gerlmaier A (2018b) Organisationale Gestaltungskompetenz im Betrieb: ein (unterschätzter) Mediator des Zusammenhangs von psychischer Belastung und Beanspruchung. Z Arb Wiss 72(2):130-136

Gerst D (2011) Sind ganzheitliche Produktionssysteme und Gute Arbeit vereinbar? Arbeit 20(3):246-250

Guillaumie L, Boiral O, Champagne J (2017) A mixed-methods systematic review of the effects of mindfulness on nurses. J Adv Nurs 73(5): 1017-1034

Hammer S, Liebherr M, Kersten S, Haas C (2015) Adherence to worksite health interventions: practical recommendations based on a theoretical framework. J Workplace Behav Health 30(4):325-343

Heaney CA, Price RH, Rafferty J (1995) The caregiver support program: an intervention to increase employee coping resources and enhance mental health. In: Murphy LR, Hurrell JJ, Sauter SL, Keita GP (Hrsg) Job stress interventions. American Psychological Association, Washington, S 93-108

Kampe J, Habenicht H, Trimpop R (2020) Erfolgreich in Kulturveränderungsprozesse starten: Vorstellung eines praxiserprobten Konzepts in KMU. In: Trimpop R, Fischbach A, Seliger I, Lynnyk A, Kleineidam N, Große-Jäger A (Hrsg) 21. Workshop Psychologie der Arbeitssicherheit und Gesundheit. Gewalt in der Arbeit verhüten und die Zukunft gesundheitsförderlich gestalten! Asanger, Kröning, S 327-330

Lechleiter P, Purbs A (2019) HR und Gesundheitsmanagement in der Arbeit 4.0. Arb Arbeitsr 1(19):30-34

Lee NK, Roche AM, Duraisingam V, Fischer JA, Cameron J (2014) Effective interventions for mental health in male-dominated workplaces. Ment Health Rev J 19(4):237-250

Leider PC, Boschman JS, Frings-Dresen MH, van der Molen HF (2015) Effects of job rotation on musculoskeletal complaints and related work exposures: a systematic literature review. Ergonomics 58(1):18-32

Maier C, Laumer S, Weinert C (2015) Enterprise resource planning systems induced stress: a comparative empirical analysis with young and elderly SAP users. Wirtschaftsinformatik Proceedings, Paper 93

Meyer SC, Tisch A, Hünefeld L (2019) Arbeitsintensivierung und Handlungsspielraum in digitalisierten Arbeitswelten - Herausfor- 
derung für das Wohlbefinden von Beschäftigten? Ind Beziehungen 26(2):207-231

Richardson KM, Rothstein HR (2008) Effects of occupational stress management intervention programs: a meta-analysis. J Occup Health Psychol 13(1):69-93

Rohrmann B (1978) Empirische Studie zur Entwicklung von Antwortskalen für die sozialwissenschaftliche Forschung. Z Sozialpsychol 9:222-245

Schaper M, Debbing C, Ruiner C, Hagemann V (2020) Entwicklung eines Risk Assessment: Beurteilung des Gefährdungspotentials von digitalisierten Arbeitsplätzen. In: Trimpop R, Fischbach A, Seliger I, Lynnyk A, Kleineidam N, Große-Jäger A (Hrsg) 21. Workshop Psychologie der Arbeitssicherheit und Gesundheit. Gewalt in der Arbeit verhüten und die Zukunft gesundheitsförderlich gestalten! Asanger, Kröning, S 327-330

Semmer NK (2011) Job stress interventions and organization of work. In: Quick JC, Tetrick LE (Hrsg) Handbook of occupational health psychology. American Psychological Association, Washington, S 299-318

Semmer NK, Zapf D, Dunckel H (1999) Instrument zur Stressbezogenen. In: Tätigkeitsanalyse ISTA, In: Dunckel H (Hrsg) Handbuch psychologischer Arbeitsanalyseverfahren. vdf Hochschulverlag, Zürich, S 179-204
Stab N, Schulz-Dadaczynski A (2017) Arbeitsintensität: Ein Überblick zu Zusammenhängen mit Beanspruchungsfolgen und Gestaltungsempfehlungen. Z Arb Wiss 71:14-25

Stummer H, Innreiter-Moser C, Moldaschl K (2008) Partizipatives Gesundheitslernen in Organisationen. Gruppendynamik 39(3):351-365

Thompson SE, Smith BA, Bybee RF (2005) Factors influencing participation in worksite wellness programs among minority and underserved populations. Fam Community Health 28(3):267-273

Warning A, Weber E (2017) Digitalisierung verändert die betriebliche Personalpolitik. IAB-Kurzbericht 12/2017. IAB, Nürnberg

Wellendorf J, Westermayer G, Riese I (2001) Die Aktion „Sicher und Gesund" der Firma Storck. In: Badura B, Litsch M, Vetter C (Hrsg) Fehlzeiten-Report 2000: Zahlen, Daten, Analysen aus allen Branchen der Wirtschaft. Zukünftige Arbeitswelten: Gesundheitsschutz und Gesundheitsmanagement. Springer, Berlin, S 231-248

Wright MT, Nöcker G, Pawils S, Walter U (2013) Partizipative Gesundheitsforschung - ein neuer Ansatz für die Präventionsforschung. Präv Gesundheitsf 8:119-121 